\title{
Generation and control of phase-locked conical wave packets in type-I seeded optical parametric amplification
}

\author{
O. Jedrkiewicz, ${ }^{1,2}$ M. Clerici, ${ }^{1,2}$ E. Rubino, ${ }^{1,2}$ and P. Di Trapani ${ }^{3, *}$ \\ ${ }^{1}$ CNISM and Department of Physics and Mathematics, Università dell'Insubria, Via Valleggio 11, 22100 Como, Italy \\ ${ }^{2}$ VINO, Virtual Institute for Nonlinear Optics, Centro di Cultura Scientifica Alessandro Volta, Villa Olmo, \\ Via Simone Cantoni 1, 22100 Como, Italy \\ ${ }^{3}$ Department of Quantum Electronics, Vilnius University, Sauletekio 9, LT 10222 Vilnius, Lithuania
}

(Received 15 April 2009; published 9 September 2009)

\begin{abstract}
We demonstrate a technique for broadband conical pulse generation based on a continuum seeded parametric amplification process in quadratic nonlinear media. We show that by using an appropriately focused broadband weak seed we are able to selectively generate and amplify a single phase-locked conical wave packet. We present experimental spatiotemporal spectra obtained with two different crystals in type I phase-matching configurations, showing the generation and amplification of single X-type and O-type wave packets, respectively, and we compare the results with the stationary wave modes that can be supported in the parametric amplification process. On the basis of numerical calculations, we also show that the broadband signal+idler phase-matched field generated by the amplifier may be characterized by a well-defined phase velocity, whose control is guaranteed by the tunability of the amplifier spectral gain curves.
\end{abstract}

DOI: 10.1103/PhysRevA.80.033813

PACS number(s): 42.65.Re, 42.65.Yj, 42.65.Sf

\section{INTRODUCTION}

Nondiffracting Bessel beams [1] and their polychromatic versions, namely, $X$ waves [2], Bessel $X$ waves [3], and Pulsed Bessel beams [4], have been intensively studied [5,6] for their potential impact for applications in optical communications, metrology, spectroscopy, and imaging [7], as well as in extreme nonlinear optics [8]. These beams and pulses are often referred to as conical waves, which are unique optical wave packets (WPs) differing from Gaussian WPs because of the presence of important off-axis (i.e., conical) energy flow. In the presence of a broad temporal spectrum, conical waves support angular dispersion, i.e., the dependence of temporal frequencies on angles. As a consequence they can propagate stationarily in linear as well as nonlinear media, in spite of diffraction and material dispersion, and exhibit tunable "effective" phase and group velocities, which results in unique dispersion-management features. Due to the mentioned degrees of freedom, conical waves emerge spontaneously in a number of nonlinear processes. Experimentally, in the presence of nonlinearity the formation of $\mathrm{X}$ waves (which are localized and propagation-invariant wave packets characterized by a hyperbolic geometry of the angular dispersion relation), has been demonstrated both in second-order $\chi(2)$ nonlinear media $[9,10]$ and in Kerr media [11-14]. Although conical WPs appear spontaneously in many nonlinear interactions, or can be created by linear optical methods (e.g., axicons, lensacons [15] and holographic masks [16]), until now the ability to efficiently generate a wide family of conical waves (CWs) has been far from being achieved.

\footnotetext{
*Permanent address: CNISM and Department of Physics and Mathematics, Università dell'Insubria, Via Valleggio 11, 22100 Como, Italy and VINO, Virtual Institute for Nonlinear Optics, Centro di Cultura Scientifica Alessandro Volta, Villa Olmo, Via Simone Cantoni 1, 22100 Como, Italy.
}

It has been theoretically shown [17-20] that the optical parametric amplifier gain, which is dictated by the energy and momentum conservation laws, i.e., the phase-matching conditions, allows the generation and amplification of localized conical WPs thanks to the angular dispersion of the phase-matched radiation. In particular, the optical parametric amplification (OPA) process is able to support the amplification of CWs that are stationary in envelope, as long as odd terms in the dispersion relation play a negligible role in defining the stationary mode spatiotemporal spectral shape; this being valid in the case of amplification over a certain (not too large) spectral bandwidth $[21,22]$. On the other hand, vacuum seeded OPA generates radiation with intriguing conical spatiotemporal coherence properties that can be described as a statistical gas of $X$ waves $[23,24]$. While the spatial mode-locking mechanism of the incoherent signal in OPA was presented in [25], the capability of obtaining a nondiffracting X-like pulsed beam, starting from the quantum-noise amplification and exploiting the self-mode-locking mechanism in the full spatiotemporal domain, has been shown in [26]. Recently, it has also been experimentally demonstrated the possibility of efficiently generating and amplifying relatively narrow-band pulsed Bessel beams, thanks to the pulse reshaping mechanism that occurs in a coherently seeded OPA [27].

In this work we propose a technique for broadband conical pulse generation based on a continuum seeded quadratic parametric amplification process in type I nonlinear crystals. We show that by using an appropriately focused broadband weak seed we are able to reshape and amplify the latter into a phase-locked conical pulse with angle-frequency spectrum supported by the parametric amplifier, and we discuss the conditions for having phase-matched WPs characterized by a well-defined global (i.e., nonlocal) phase velocity. It is worth recalling that the ability of modifying the refractive index seen by the extraordinarily polarized wave propagating in the nonlinear crystal, or in other words the ability of changing 
the pump phase velocity, reflects in the possibility of tuning the angular dispersion of the amplifier gain (i.e., by changing the angle or temperature of the nonlinear crystal the phasematching curves change). Here we show that this property renders the nonlinear reshaping mechanism unique in terms of flexibility and control of the phase velocity of the conical wave modes generated in OPA. Moreover by optimizing the phase-matching conditions we illustrate the possibility of generating experimentally single X-type or O-type conical waves $[21,22]$, and we discuss their stationarity through a comparison with the localized wave modes supported by the dispersive material.

The paper is organized as follows. In Sec. II, we present the experimental results obtained with a type I beta barium borate $(\mathrm{BBO})$ crystal in a continuum seeded OPA process pumped at $352 \mathrm{~nm}$, which leads to the generation around degeneracy of broadband amplified radiation in the normal group velocity dispersion regime and thus to the observation of tunable X-type waves. On the other hand, in Sec. III we illustrate the case of continuum seeded OPA based on a temperature tuned type I lithium triborate (LBO) crystal pumped at $527 \mathrm{~nm}$. In this regime, the broadband amplified radiation is widely extended also in the anomalous group velocity dispersion region thus supporting the creation of tunable O-type waves. In both cases we present experimental spatiotemporal spectra of the amplified radiation emitted in different phasematching conditions, and in the case of the $\mathrm{X}$ waves we show that the total field generated in the phase-matched OPA process may be characterized by a well-defined phase velocity that turns out to be equal to that of the pump. We also illustrate how with a proper spectral tailoring, we are able to generate conical pulses which can be defined within a good approximation as stationary modes of the dispersive material. An analysis of the spatiotemporal near-field pattern obtained as Hankel-Fourier transform of the spectra recorded is also made under the assumption of flat phase, illustrating the possibility of obtaining ultrashort and localized coherent conical wave packets. A discussion and conclusions are presented in Sec. IV.

\section{X-TYPE WAVE PACKET GENERATION}

\section{A. Experimental results}

The first OPA experiment was realized by pumping at 352 $\mathrm{nm}$ a $2 \mathrm{~mm}$ type I beta barium borate crystal and by seeding the latter around degeneracy with a strongly focused broadband radiation obtained from the supercontinuum generation mechanism occurring in a Kerr medium. More precisely we have used the technique proposed in [28] leading to the formation of a strongly blueshifted, spectrally isolated radiation associated with the collapse of a $1.2 \mathrm{ps}, 1055 \mathrm{~nm}$ pump pulse in bulk fused silica in the normal group velocity dispersion (GVD) regime. The continuum radiation generated ranges approximately between 400 and $700 \mathrm{~nm}$, thus covering half spectral range of the amplifier gain bandwidth, which we shall refer here as the signal region.

In the experimental setup, the $1055 \mathrm{~nm}, 1.2 \mathrm{ps}$ input beam, delivered by a $2 \mathrm{~Hz}$ amplified Nd:glass laser (Twinkle, Light conversion Ltd.) was divided into two by means of a
30:70 Beam Splitter. One arm was used for third harmonic generation, obtaining the $352 \mathrm{~nm}$ carrier wavelength pulse used as pump of the OPA process (beam dimension at the crystal input was approximately $200 \mu \mathrm{m}$ [full width at half maximum (FWHM)]. The beam in the other arm was focused on a $2 \mathrm{~cm}$ long fused silica sample, by means of a 50 $\mathrm{cm}$ focal length lens, for the generation of the broadband continuum as described just above. The generated radiation was collimated and then refocused inside the BBO crystal by means of a $30 \mathrm{~cm}$ and a $6 \mathrm{~cm}$ focal length lenses, respectively, reaching a beam transverse dimension of few micrometers. Seed $(30 \mathrm{~nJ})$ and pump $(50 \mu \mathrm{J})$ pulses were then collinearly recombined by means of a beam splitter (High Transmittivity mirror @ $400 \mathrm{~nm}$ ) in the $2 \mathrm{~mm}$ long type I BBO crystal. Note that the strong focusing conditions of the seed guaranteed a sufficiently broad distribution of the spatial spectrum to cover all the angular bandwidth of the OPA. On the other hand, although the exact temporal and chirp characteristics of the used seed were unknown, a delay line permitted to control the temporal superposition and best interaction of the pump and seed pulses inside the nonlinear medium, from the intensity maximization of the visible radiation emitted from the crystal. Indeed when the two pulses spatially and temporally overlap inside the OPA crystal, the broadband seed is amplified over a wide range of frequencies. This can be appreciated in Fig. 1 where a single-shot image of the amplified spatiotemporal angular spectrum in the $(\theta, \lambda)$ domain has been recorded by a modified NIKON camera (with the infrared filter removed). As expected, although the seed spectral range does not extend beyond the degenerate wavelength $(704 \mathrm{~nm})$, the amplification of the phase-matched spectral components of the radiation occurs thanks to the phase-conjugation process over the entire recorded bandwidth of the amplifier. In fact in the OPA process, whenever a signal frequency component is amplified, an idler frequency component satisfying the energy and momentum conservation law is also automatically generated and amplified. In these experimental conditions we were thus able to record simultaneously signal and idler in one single shot, evidencing the formation of a single X-type wave over the entire amplified bandwidth, which is as wide as $450 \mathrm{~nm}$.

The nature of the generated radiation, in terms of its spatiotemporal modes structure, was more precisely investigated from a quantitative study of the measured far-field spatiotemporal $(\theta, \lambda)$ spectrum, in analogy with the analysis performed in $[23,24]$. The diagnostics technique (see [12] for details) was based on an imaging spectrometer (MS 260i, Lot-Oriel) combined with a 16 bit high efficiency charge-coupled device (CCD) camera (Andor). The possibility to tune the phase-matching curves of the nonlinear BBO crystal, i.e., the spatiotemporal gain characteristics of the OPA process, allows us to experimentally control features and dispersion characteristics of the generated conical wave packets. In order to illustrate this idea we have analyzed the parametric amplification process in different phase-matching conditions around the degenerate frequency. In particular these correspond to three different values of the crystal tuning angle $\theta_{t}$, i.e., angle between the input pump pulse direction and the crystal optical axis, namely $\theta_{t}=33^{\circ}, 32.61^{\circ}$, and $32.50^{\circ}$.

Figure 2 shows the comparison between the single-shot spectral acquisitions of the parametric down-conversion ra- 


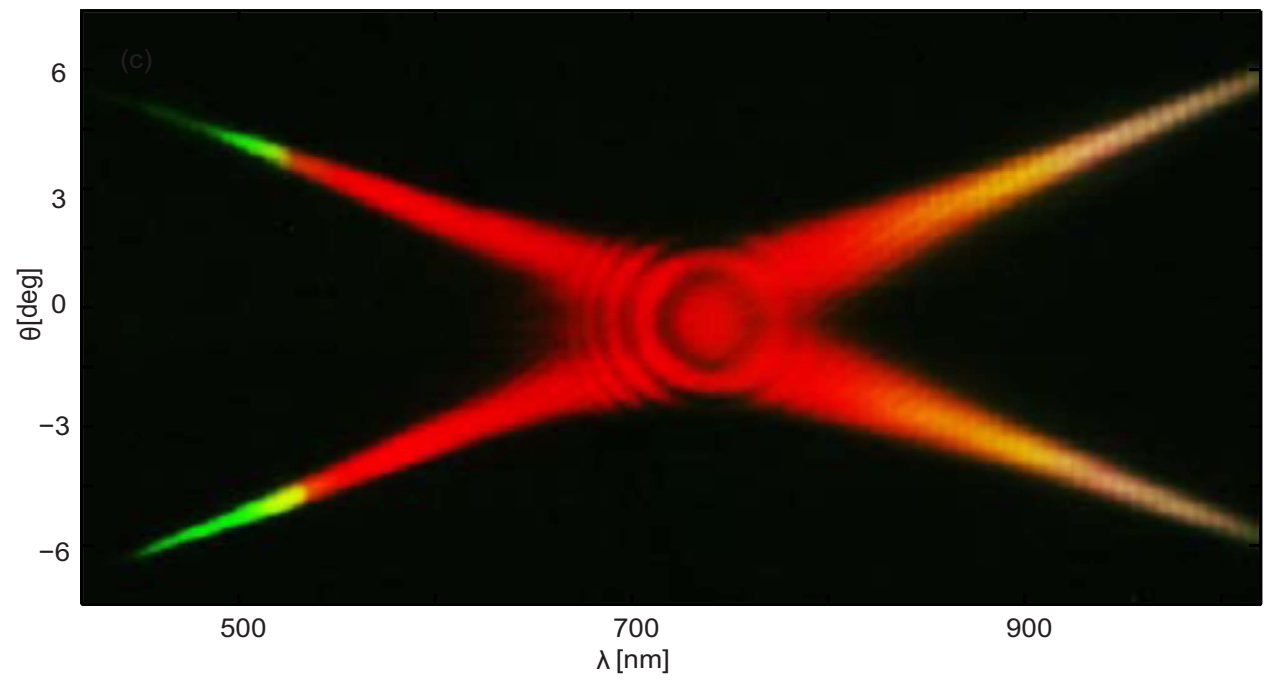

FIG. 1. (Color online) Singleshot $(\theta, \lambda)$ spectrum of the amplified radiation generated in a continuum seeded $2 \mathrm{~mm}$ long type I BBO crystal pumped at $352 \mathrm{~nm}$, and recorded by means of a modified digital NIKON camera.

diation generated in nonseeded configuration (left images) and the corresponding single-shot images recorded in the regime of parametric amplification of the broadband seed (right images). As mentioned above, the parametric downconverted radiation is generated along the so-called gain or phase-matching curve, satisfying the energy and momentum conservation laws. Note that when there is no seed (cases a, $\mathrm{c}$, and e), as a consequence of the fact that the OPA process
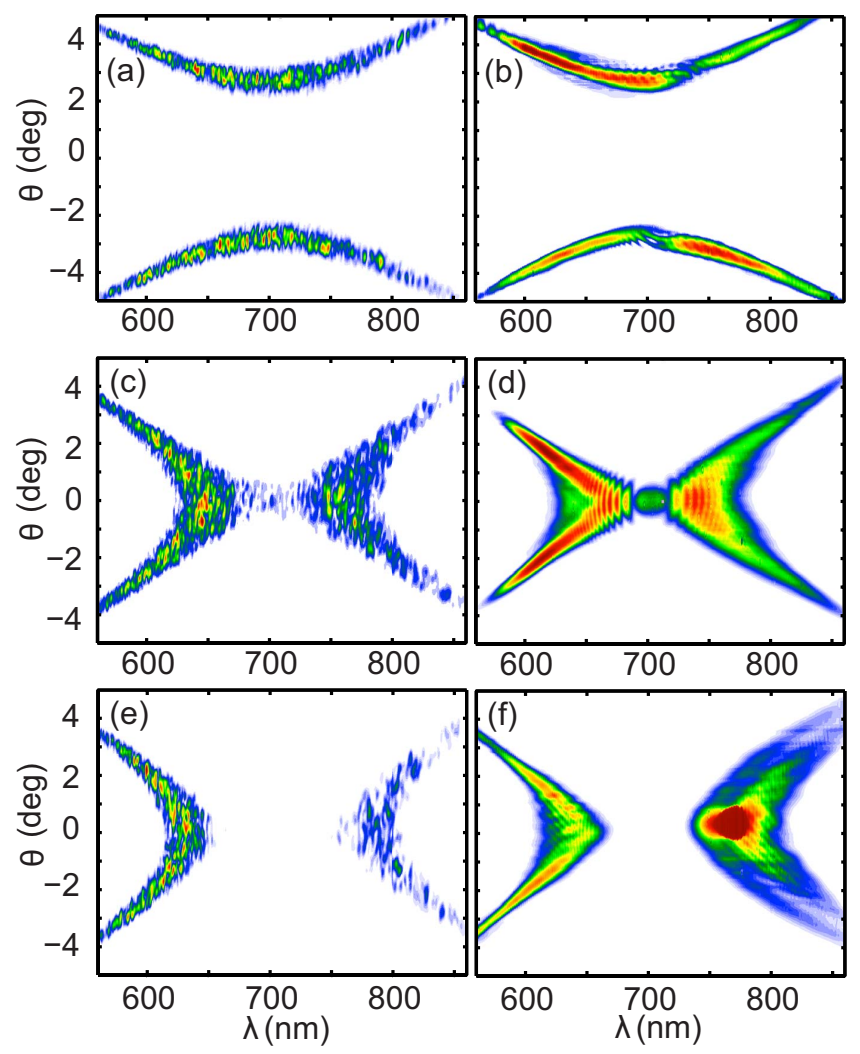

FIG. 2. (Color online) Single-shot $(\theta, \lambda)$ spectra of the radiation generated in a $2 \mathrm{~mm}$ long type I BBO crystal recorded in nonseeded (left) and continuum seeded (right) configurations, for three different phase-matching conditions. [(a) and (b) $] \theta_{t}=33^{\circ},[(\mathrm{c})$ and (d)] $\theta_{t}=32.61^{\circ}$, and $[(\mathrm{e})$ and (f) $] \theta_{t}=32.50^{\circ}$. amplifies the quantum vacuum fluctuations, the far-field spectra are characterized by speckles that fluctuate from shot to shot, also indicating the multimode nature of the generated field. When the broadband phase-locked continuum seed is injected inside the BBO crystal and the temporal superposition is guaranteed, the amplification process occurs over the same broadband region dictated by the OPA gain curve, with the difference that in this case the recorded single-shot spatiotemporal spectra are characterized by a smoothed, nonspeckle-like, structure, identical from shot to shot. We measured the energy of the amplified radiation to be about $1.30 \mu \mathrm{J}$, thus corresponding, given the seed and pump input energies of $30 \mathrm{~nJ}$ and $50 \mu \mathrm{J}$, respectively, to a gain factor of the OPA process of about 40 .

\section{B. Phase velocity, stationarity, and localization of the X-type waves generated in type I OPA}

The X-type waves generated and illustrated in the preceding section have the characteristics of being composed simultaneously by two conjugated fields (signal and idler) linked to the pump field by a well-defined phase relation, coming from the phase-matching conditions of the parametric amplification process (energy and momentum conservation laws). We shall see here that we can introduce the concept of phase velocity for the whole X-type conical wave that is amplified in the OPA process around degeneracy. For this purpose we can evaluate the properties of the generated total phase-matched field, propagating in the nonlinear medium along the $z$ direction. The propagation we consider is the linear propagation. For the sake of clarity we shall consider the projection of the field along the propagation direction, discarding the transverse spatial coordinates. The total field thus reads $E(z, t)=E_{s}(z, t)+E_{i}(z, t)$, where $E_{s}(z, t)$ $=\int d \omega A(\omega) \exp \left\{i\left[k_{s, z}(\omega) z-\omega t\right]+\phi_{s}(\omega)\right\} \quad$ and $E_{i}(z, t)$ $=\int d \omega A(\omega) \exp \left\{i\left[\left[k_{p}-k_{s, z}(\omega)\right] z-\left(\omega_{p}-\omega\right) t-\frac{\pi}{2}-\phi_{s}(\omega)\right]\right\} \quad$ are, respectively, the signal and the idler fields along $z$, having assumed equal amplitudes, i.e., $A_{s}(\omega)=A_{i}(\omega) \equiv A(\omega)$. Note that this condition is well satisfied in our case where the injected broadband seed is very weak. Clearly the phase relation involved in the three wave mixing process and used 
above is $\phi_{i}+\phi_{s}+\frac{\pi}{2}=\phi_{p}$, where for simplicity we have put the phase of the pump equal to zero. By using the phaseconjugation properties of the parametric amplification process, we obtain, after a few calculational steps, the expression for the generated field that can be written as a product of a phase term and a real quantity as

$$
\begin{aligned}
E(z, t)= & \exp \left[-i\left(\frac{k_{p}}{2} z-\frac{\omega_{p}}{2} t-\frac{\pi}{4}\right)\right] \int d \omega A(\omega) \\
& \times \cos \left[\left(k_{s, z}(\omega)-\frac{k_{p}}{2}\right) z-\left(\omega-\frac{\omega_{p}}{2}\right) t+\phi(\omega)+\frac{\pi}{4}\right]
\end{aligned}
$$

The real part of the total phase-matched field is thus given by

$$
\begin{aligned}
\tilde{E}(z, t)= & \cos \left[\frac{k_{p}}{2}\left(z-v_{p} t\right)-\frac{\pi}{4}\right] \int d \omega A(\omega) \\
& \times \cos \left[\left(k_{s, z}(\omega)-\frac{k_{p}}{2}\right) z-\left(\omega-\frac{\omega_{p}}{2}\right) t+\phi(\omega)+\frac{\pi}{4}\right]
\end{aligned}
$$

where $v_{p}$ is the phase velocity of the pump field.

Interestingly, from this analysis it turns out-and it will also be clarified below-that the field describing the conical WP generated in the OPA process, composed by both the signal and idler and propagating linearly along the $z$ (i.e., pump) direction, evolves with a well-defined phase velocity equal to that of the pump field. It should thus be characterized by having, in the reference frame of the pump phase velocity, its zeros in fixed positions. Note that the integral term in Eq. (2) leads to an amplitude modulation, which is typically much slower than that of the carrier.

Let us now illustrate the result that with the phasematched conical wave packets supported by the amplifier we can associate the physical concept of global and thus nonlocal-phase velocity. To this end, we report in Fig. 3(a) a plot of the total (signal plus idler) real field generated in OPA in the conditions of X-type phase matching, as function of the propagation $z$ (linear propagation) and of its proper time evolution $t^{\prime}$. Note that we consider linear propagation in the plane-wave approximation and dispersion at all orders, i.e., introducing in the propagation code the full Sellmeier relation of the BBO crystal. More precisely, we have considered a nearly single cycle, transform limited pulse seed centered around $650 \mathrm{~nm}$, and the same phase-matching conditions as in Fig. 2(c). If the reference frame chosen to plot the field is the one traveling at a velocity $v_{r e f}$, it is related to the laboratory reference frame by the relations $z^{\prime}=z$ and $t^{\prime}=t$ $-z / v_{\text {ref }}$. It can also be shown that in the moving reference frame, the local-phase velocity $v_{p h}$ of a portion of the field wavefront is linked to $v_{r e f}$ by $1 / v_{p h}=1 / v_{r e f}+d t^{\prime} / d z$. In the case of Fig. 3(a), the velocity $v_{\text {ref }}$ of the moving reference frame, used to plot the field, is the phase velocity of the pump, i.e., $v_{r e f}=v_{p}$. In Fig. 3(b), we report for comparison the evolution of the real part of the field of a Gaussian wave packet of equivalent bandwidth, in the reference frame of its own carrier frequency phase velocity. By looking at Fig. 3(a) we can observe that the zeros of the plotted field are fixed
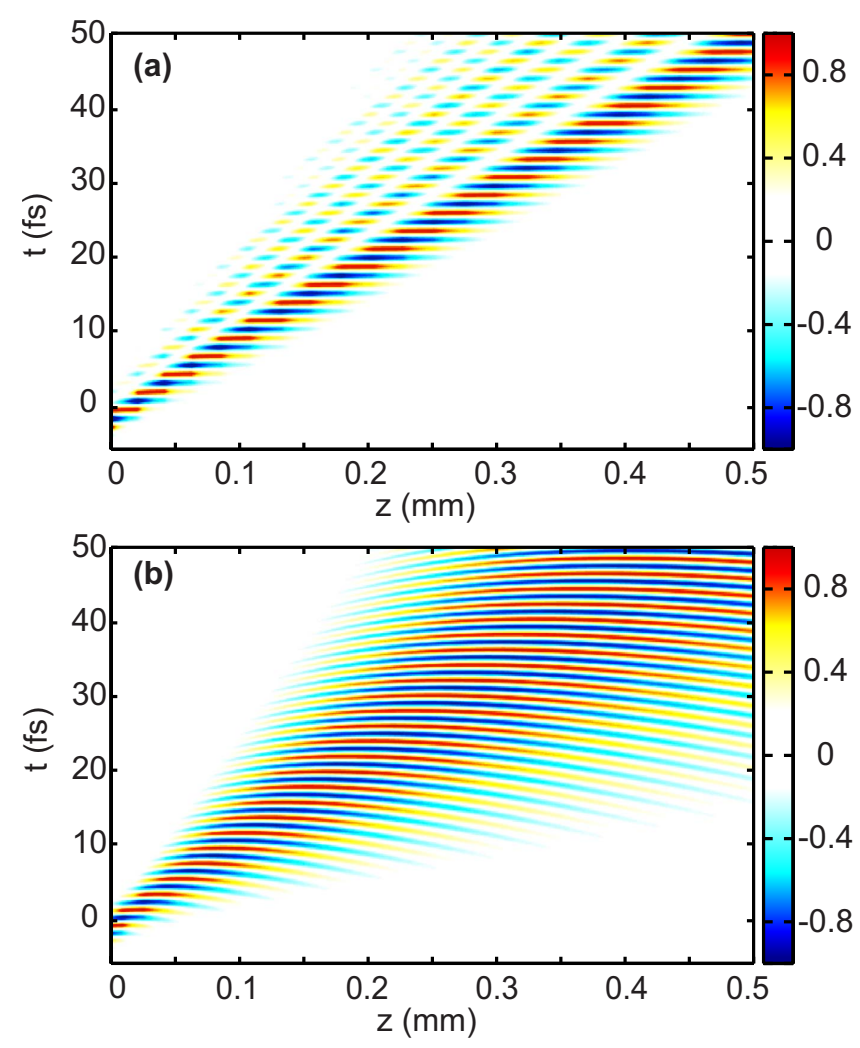

FIG. 3. (Color online) Evolution along $z$ of the on-axis temporal profile of (a) the signal+idler field in condition of X-type phasematching, as in Fig. 2(c); (b) evolution of a Gaussian WP of equivalent bandwidth. The reference frame is, respectively, (a) the one of the pump phase velocity, and (b) the Gaussian WP carrier frequency phase velocity reference frame. Note the subdispersive behavior of the WP in (a), characterized by third order dispersion contribution in comparison with the Gaussian WP typical dispersion clearly shown in (b). Also note that in (a) a field wavelength appears to be well defined as a nonlocal property, differently from (b) where the chirp prevents from having a global definition of phase velocity.

and independent of the propagation position $z$ (by slicing the plot at several $z$ positions, these zeros are fixed in time). Therefore the presence of perfectly horizontal lines in the figure confirms the fact that we can introduce, for the X-type WP generated in OPA, the concept of non-local-phase velocity. The total phase-matched signal+idler field propagating along $z$ is thus characterized by a global phase velocity that is locked (being everywhere equal to that of the pump), and is certainly different from the typical definition of the phase velocity usually associated with each monochromatic components of a standard wave packet. Indeed we can see that, in contrast, in the case of the Gaussian WP plotted in Fig. 3(b), we can only define a local-phase velocity (of each zero of the field) that clearly varies along the pulse. In particular for a given position $z$ we can evidence the portion of the leading part of the Gaussian pulse (redshifted components) where $v_{p h}>v_{r e f}$, and the portion of the pulse trailing part (blueshifted components) where $v_{p h}<v_{r e f}$. In the two plots of Figs. 3(a) and 3(b), the envelope modulation of the field travels with the WP group velocity, while we can observe the spreading of this modulation due to dispersion. The X-type 
wave packet selected and amplified in the OPA process is subdispersive as it clearly appears from the comparison with the Gaussian WP propagation properties. On the other hand note in Fig. 3(a) the development, along the propagation direction, of an amplitude modulation. The particular, asymmetric shape of this modulation is the effect of the third order dispersion, which is not compensated by the angular dispersion induced by the phase-matching requirement. Thus the WP does not possess a stationary profile.

In Fig. 4, we compare the evolution of the total (signal plus idler) field [Fig. 4(c)] with the evolution of the signal field [Fig. 4(a)] and idler field [Fig. 4(b)] taken separately, in the reference frame of the pump phase velocity. In this case, we have considered an arbitrary chirp of the seed, by introducing a fourth-order phase relation in the frequency domain, with arbitrary parameters. Note therefore that here, in contrast to the case of Fig. 3(a) (transform limited wave packet), the total field at $z=0$ is characterized by a strong phase modulation. We can see that only in the case of a WP composed by the signal+idler contributions generated and amplified in the OPA, we can speak about a well-defined non-local-phase velocity of the field, which is equal to that of the pump. The fact that the conical pulse generated is chirped does not affect this property. Note on the other hand that the integral term of Eq. (2) gives rise in this case [Fig. 4(c)] to a modulation much larger than the one of Fig. 3(a), evidencing here the occurrence of a clear pulse splitting.

The fact that the phase-matched conical wave packet generated in OPA presents a clear subdispersive property, as it has appeared from the theoretical analysis presented above, permits us to study the analogy with the so-called stationary modes of the nonlinear material [21]. In general, it can be shown that in the nonparaxial approach, stationary wave modes may be introduced in the spatiotemporal frequency domain as localized waves, for which the axial component $k_{z}$ of the wave vector of the various monochromatic and planewave constituents varies linearly with frequency [29], i.e., $k_{z}(\Omega)=k_{0}-\beta+\left(k_{0}^{\prime}-\alpha\right) \Omega$, with $\alpha$ and $\beta$ being free parameters, and $k_{0}$ being associated with an optical frequency $\omega_{0}$ of the wave packet. A description of wave modes in dispersive media can thus be made through the transversal dispersion relation $K=\sqrt{k_{0}^{2}(\omega)-k_{0, z}^{2}(\omega)}$. By adjusting opportunely the values of $\alpha$ and $\beta$, the dispersion relation curve can then be compared with the one describing the phase-matching process in the nonlinear medium under consideration. Note that Orlov and co-workers have demonstrated within a paraxial approximation approach that the creation of broadband localized fields inside the crystal of optical parametric generators is feasible [17].

In this work, the possibility to generate experimentally, within a well determined spectral bandwidth, stationary X-type modes of the radiation is demonstrated in Fig. 5, where the calculated phase-matching curves and the wave mode stationary curves of the radiation in the BBO crystal have been plotted for comparison in different conditions, over the recorded experimental spectra. More precisely, we have considered three phase-matching angles where the generation of X-type conical WPs in seeded OPA has been shown (see Fig. 2), and different sets of parameters $\alpha$ and $\beta$.
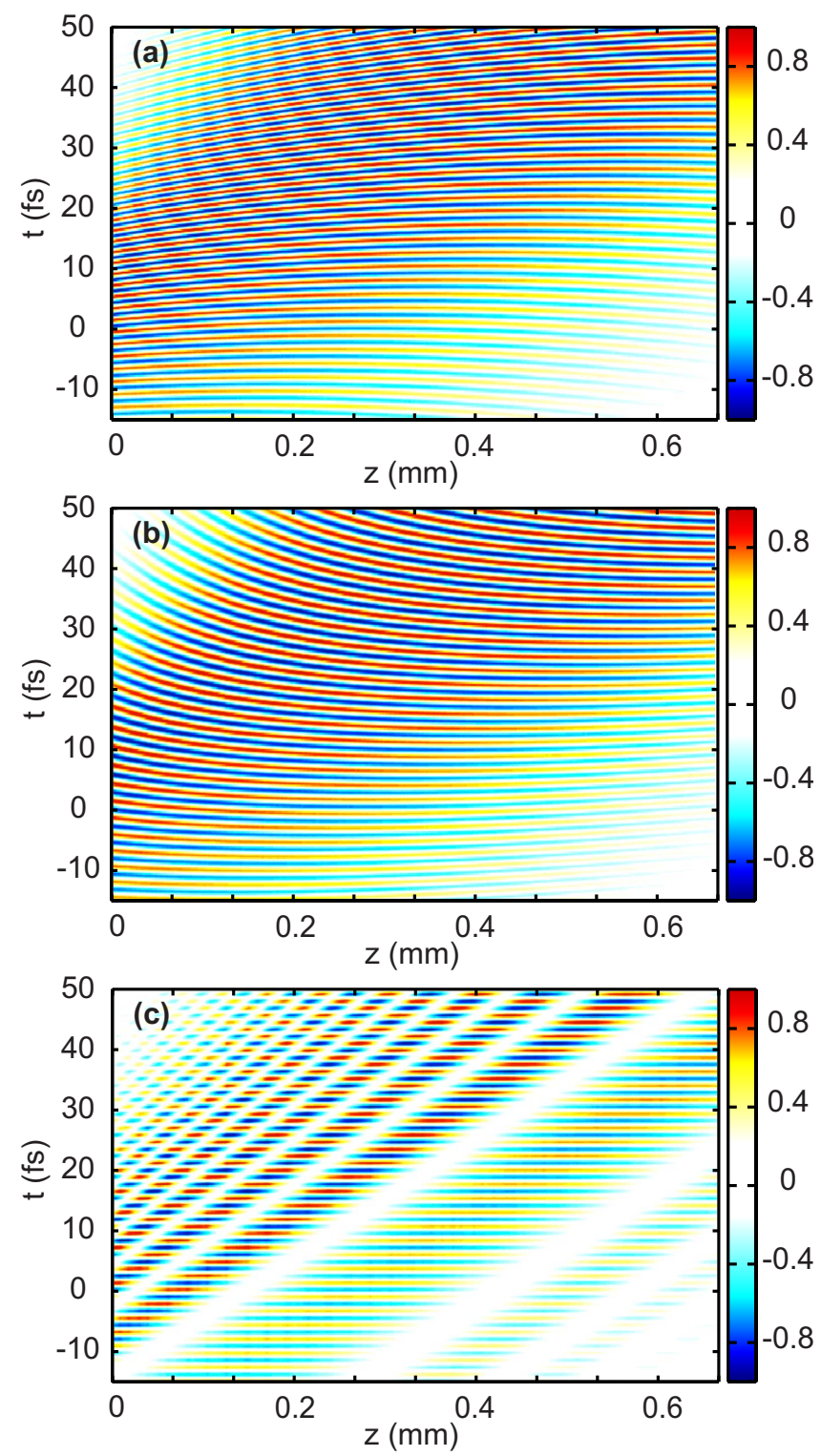

FIG. 4. (Color online) Evolution along $z$, in the pump pulse phase velocity reference frame, of the on-axis temporal profile respectively of (a) the signal, (b) the idler and (c) the signal+idler field, in condition of X-type phase matching as in Fig. 2(c). The signal pulse is characterized by a nearly single cycle transform limited bandwidth and by an arbitrary chirp. Following from phasematching conditions the idler field is also chirped. While the signal and the idler field taken separately do not show a defined global phase velocity, the total field (signal+idler) shows a clear temporal modulation, namely, the field wavelength, which is constant and global. This guarantees the possibility of defining a phase velocity for the phase-matched WP, which turns out to be the pump phase velocity.

Note that in the case of Fig. 5(c), good agreement between phase-matching and stationary conditions can only be reached independently for the signal or for the idler alone. We also mention that the stationarity requirement is equivalent to the phase-matching conditions only in the temporal quadratic approximation, thus suggesting the possibility to 

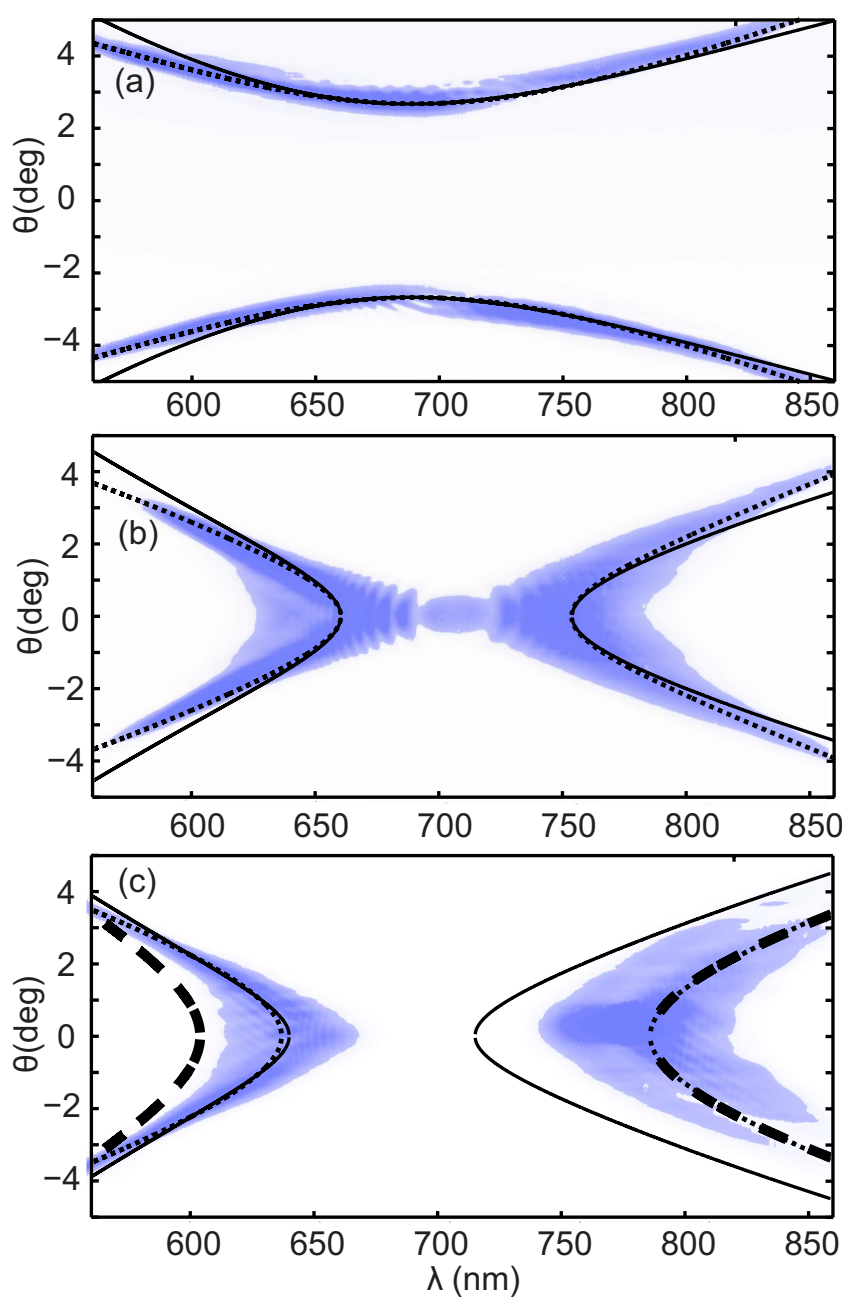

FIG. 5. (Color online) Comparison between a portion of phasematching curve (dotted curve) and the spatiotemporal spectrum of the stationary wave mode in BBO (solid or dashed curve) for three different phase-matching conditions and different sets of values of the stationary curves parameters $\alpha$ and $\beta$. In (a) $\theta_{t}=33^{\circ}, \alpha=-3$ and $\beta=6$, in (b) $\theta_{t}=32.61^{\circ}, \alpha=-0.2$ and $\beta=-1.32$, in (c) $\theta_{t}=32.5^{\circ}$ and $\alpha=-8$ and $\beta=-5.5$ (dashed curve); $\alpha=-10$ and $\beta=-0.5$ (solid curve)

obtain stationary WPs when a suitable spectral windowing is made for instance on the input seed.

We observe that the X-type wave illustrated in Figs. 5(a) and 5(b), respectively-made up of signal and idler simultaneously - can be well approximated by a stationary mode of the material, and from the considerations made previously we can also say that the generated WP should be characterized by a field having a phase velocity equal to that of the pump. To this end it is interesting to note that for localized wave modes associated with transform limited pulses and featured by having a regular symmetrical spectrum centered around its "gravity center," Porras and coworkers introduced the local concept of "effective" phase velocity for the core of the wave mode (i.e., around the peak of the pulse) [22]. Nevertheless such a quantity differs from the non-local-phase velocity defined above at which all the zeros of the OPA WP field travel, and which is well defined and constant across the whole pulse profile and along propa- gation. On the other hand, in [22] it was also shown that if the spectrum is centered around the degeneracy frequency $\omega_{s}=\omega_{i}=\omega_{0}$ (as in the case of the $\mathrm{X}$ waves), this effective phase velocity reduces to $v_{p h}=\omega_{0} /\left(k_{0}-\beta\right)$ describing both the core, and the leading and trailing parts of the wave mode [22], and thus approaches, although being different, the concept of global phase velocity that has just been introduced in the present paper.

From the values of the parameter $\beta$ used in Figs. 5(a) and 5 (b) to obtain the stationary mode approximating the OPA gain curve, we find that the ratio: $\left(v_{p h}-v_{p}\right) / v_{p}$ is of the order of $10^{-5}$. Clearly, it appears from this result that in the case where the spectrum contains both the signal and idler contributions of the field generated in the OPA process, and where it can be approximated by a stationary wave mode of the dispersive material, the phase velocity of the X-type WP can be directly extracted from a fit of the dispersion relation of the spectral curve. Moreover, it turns out that thanks to the tunability of the spatiotemporal gain curve of the OPA process, the generation of stationary conical waves with different phase velocities becomes therefore controllable. In particular we mention the possibility to generate WPs with either luminal or subluminal phase velocity, which is of great interest for the optimization of phase-matching related nonlinear problems.

Interestingly, going back to Fig. 2(d) we can observe in the angular spectrum of the amplified radiation an on-axis fringe pattern. This modulation appears under the form of concentric rings around degeneracy and is due to the phasesensitive optical parametric amplification process, which leads to the generation of signal and idler related by a welldefined phase relation, as seen precedently. Indeed the phase matching will select and amplify only those spectral components satisfying the latter. Note that this occurs both in the spatial and in the temporal domain and gives rise to an interference pattern which depends on the mutual phase relation. In particular if the spatial and the temporal phases have the same sign (meaning for example that both are positively chirped), then the interference pattern will be made by concentric rings; otherwise it will be "X shaped." In turn supposing fixed the temporal chirp of the continuum seed, the concentric fringe pattern appearing in Fig. 5(d) is due to the interference process between the amplified injected field and idler field generated during the amplification, and thus has its origin in the spatial phase of the focusing input seed pulse. Indeed the particular shape of these fringes can be varied and analyzed by changing the focusing conditions of the input continuum seed. As an illustration of this phenomenon we show in Fig. 6 the spatiotemporal power spectra of the amplified radiation generated in the collinear configuration in three different cases associated, respectively, with three different positions of the $\mathrm{BBO}$ crystal with respect to the seed focus. The first image been recorded with the BBO placed 2 $\mathrm{mm}$ before the focus position and thus corresponding to a converging input seed [Fig. 6(a)], the second has been recorded with the $\mathrm{BBO}$ placed at the focal plane of the seed focusing lens [Fig. 6(b)], and thus corresponding to an input seed with a flat curvature in the waist, and finally the last recorded image has been taken with the BBO placed $2 \mathrm{~mm}$ after the focus, thus corresponding to an input seed with a 

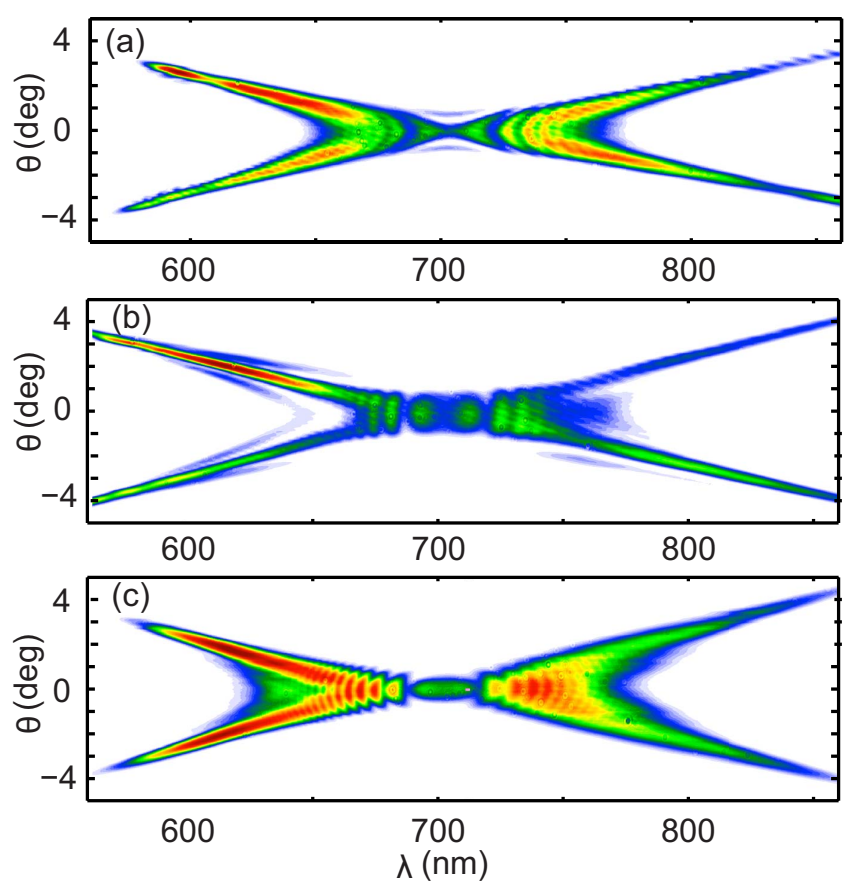

FIG. 6. (Color online) Single-shot amplified signal spectrum for $\theta_{t}=32.68^{\circ}$ for three different positions of the BBO crystal with respect to the seed focus: (a) before the focus, (b) approximately in the focus, and (c) slightly after the focus.

diverging spatial phase [Fig. 6(c)]. We mention that in our experimental conditions, the Rayleigh range of the seed beam has been estimated to be about $50 \mu \mathrm{m}$. Different interference patterns can be clearly observed in the three cases. Note that a similar effect is expected to occur if the temporal phase of the seed is varied. For instance in the case of a transform limited seed pulse (no chirp) we could expect to have a power spectrum presenting no interference fringes along the temporal frequency axis.

We can evaluate the coherence volume associated with the emitted signal or idler radiation by means of WienerKhinchin theorem, in analogy with the study presented in $[23,24]$. The space-time coherence function retrieved can give us an insight of the near-field structure in the $(r, t)$ space, where $r$ is the radial coordinate in the transverse plane, of the reshaped and amplified WP in the OPA process, under the assumption of transform limited conditions (maximum spatiotemporal localization achievable). The spatiotemporal amplitude profile plotted in Fig. 7 has been numerically evaluated by means of an inverse Hankel-Fourier transform of an averaged spatiotemporal power spectrum [recorded in conditions of Fig. 2(b)]. Noting that the amplification process is more efficient in the case of seed pulse with zerocurvature in the waist, the Hankel-Fourier transform has been performed under the assumption of flat temporal and spatial phases. Although this condition might not be satisfied in our experiment, the result allows us to have an indication of the limit achievable, showing thus the possibility to amplify a temporally and spatially well localized $\mathrm{X}$ wave with a central peak characterized by a duration and a transverse size of a few femtoseconds and microns, respectively. This result opens the possibility to generate transform limited WPs char-

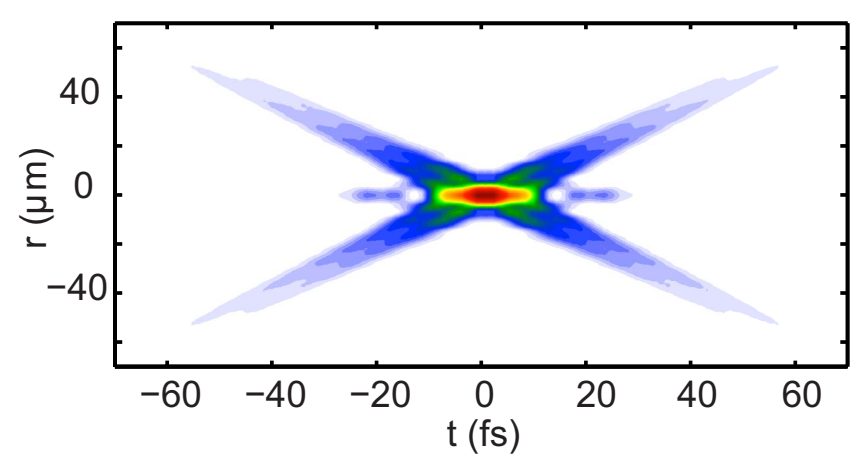

FIG. 7. (Color online) Numerical evaluation of the near-field amplitude profile associated with the angular spectrum of Fig. 2(b), under the assumption of flat spatial and temporal phases.

acterized by a highly localized near-field intensity profile, by suitably shaping the spectral phase (chirp) of the input continuum seed of the OPA.

\section{O-TYPE WAVE PACKET GENERATION}

\section{A. Experimental results}

We have seen in the preceding section that if the OPA is coherently seeded, a conical pulse can be generated thanks to the reshaping and selective amplification mechanism occurring along the excited phase-matching curve. In the second experiment, we have exploited this opportunity to generate a different type of conical wave, namely the so-called O-type wave. This result was achieved with a temperature tuned, Type I, $30 \mathrm{~mm}$ long LBO crystal pumped with a $200 \mathrm{fs}, 527$ $\mathrm{nm}, 20 \mu \mathrm{J}$ pulse (compressed second harmonic of Twinkle laser pulse, Light Conversion Ltd.) and seeded by a broadband weak radiation. The latter was obtained by spectral windowing a filament induced by focusing in a $5 \mathrm{~cm} \mathrm{BK7}$ bulk sample a 1 ps laser pulse at $1055 \mathrm{~nm}$ (Twinkle first harmonic). The continuum seed pulse generated over a smooth bandwidth in the range 600-800 $\mathrm{nm}$, with energy in the $\mu \mathrm{J}$ range was then strongly focused with a $5 \mathrm{~cm}$ lens and injected collinearly with the pump inside the nonlinear crystal. Note that in the chosen configuration of OPA, which was tuned for operation well out of degeneracy, only the high frequency region (visible range) of the amplifier spectral gain curve was seeded. As before, thanks to the strong focusing conditions of the seed, we could guarantee an adequate distribution of its transverse wave vectors compatible with the phase-matched spatial frequencies of the OPA. Here again the temporal overlap of the pump pulse and seed pulse inside the crystal was controlled by means of a delay line.

Figure 8 shows the single-shot spatiotemporal spectra of the signal radiation recorded in nonseeded and seeded configuration, respectively, and for two different crystal temperatures (i.e., phase-matching conditions) both corresponding to emission out of degeneracy, chosen for a best superposition range with the spectral characteristics of the continuum. Note in the left images the typical specklelike pattern reflecting the incoherent nature of the parametric downconversion (PDC) radiation. Indeed, as already observed in the first experiment described in Sec. II, in the 

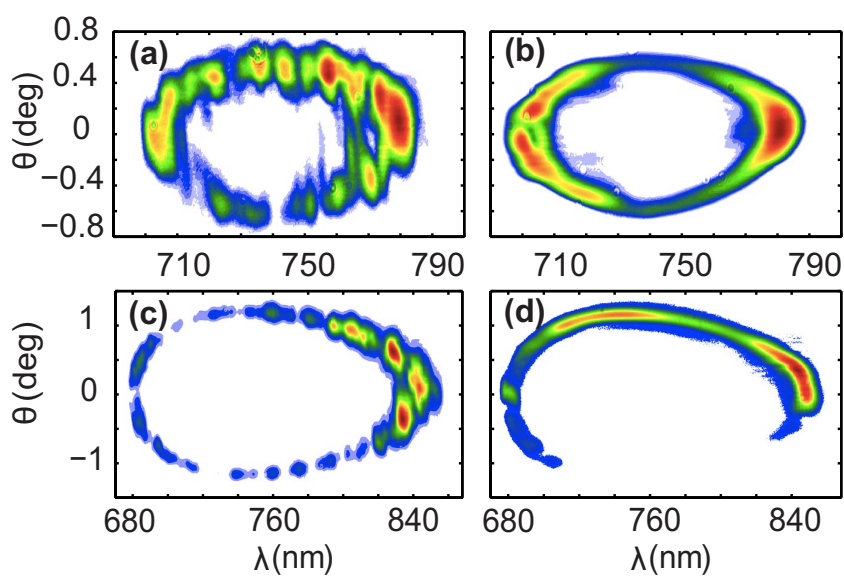

FIG. 8. (Color online) Single-shot spatiotemporal spectra of the parametric radiation generated in OPA recorded at the output of a $30 \mathrm{~mm}$ type I LBO crystal for two different crystal temperatures, in nonseeded [(a) $T=120^{\circ}$ and (c) $T=143^{\circ}$ ] and continuum seeded [(b) $T=120^{\circ}$ and (d) $T=143^{\circ}$ ] configurations, respectively.

nonseeded case this specklelike pattern of the radiation spectrum is different from shot to shot of the pump pulse. Because of the phase-conjugation property of the amplified signal and idler radiation in OPA, the same mechanism has to occur also for the idler, which is generated through phasematching in the infrared region, with the same statistical properties as the signal. Even if the continuum seed spans only the visible-near infrared (e.g., 600-800 nm) bandwidth, smooth O-type single-shot spectra of the idler were indeed separately observed with a different diagnostics in the seeded OPA configuration (data not shown).

\section{B. Stationarity and localization of the O-type waves generated in type I OPA}

In this experiment, where the crystal was tuned well out of degeneracy, and where we were interested only in the detection of the amplified signal radiation in the visible range, the concept of field phase velocity cannot be applied, as discussed in the preceding section, although it could in principle be defined when considering simultaneously both the visible and far infrared radiation of signal and idler. On the other hand we shall concentrate here on the properties of stationarity of the O-type waves generated. Indeed, interestingly the O-type spatiotemporal spectral distribution is characteristics of the localized wave modes called O-waves, which are stationary modes in materials with anomalous group velocity dispersion [21], i.e., for $\lambda>1 \mu \mathrm{m}$ in LBO (idler region).

Figure 9 illustrates the bandwidth over which the exact phase-matching curves associated with the infrared idler field match in a good approximation the stationary modes of the $\chi^{(2)}$ crystal, analytically computed by suitably setting the parameters $\alpha$ and $\beta$ defined before. We point out again that the fact that the correspondence between the phase-matching conditions and the stationary spectral modes is restricted to a limited bandwidth, implies experimentally the necessity of a spectral windowing process in order to obtain a WP satisfy-
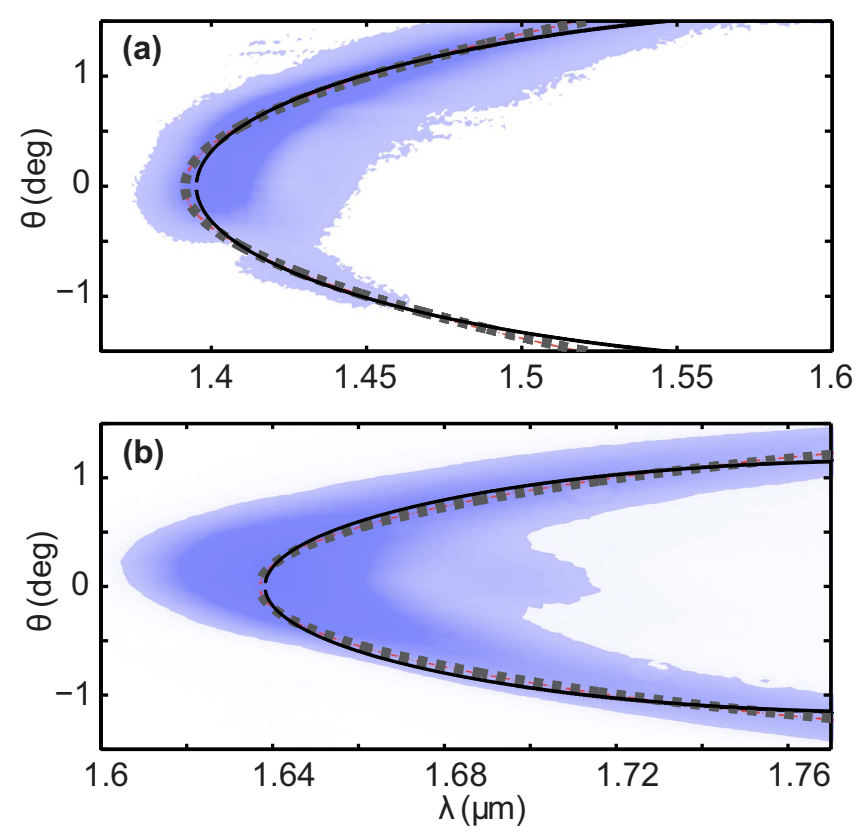

FIG. 9. (Color online) Comparison between phase-matching curves (black solid lines) and stationary $\mathrm{O}$ waves dispersion curves (black dashed) supported by the LBO crystal in different cases. (a) $T=120^{\circ}, \quad \alpha=-29.5$ and $\beta=-18.4$, (b) $T=143^{\circ}, \alpha=-10.7$ and $\beta=-5.1$. The blue pattern underneath the curves illustrates the expected idler spatiotemporal spectral distribution, numerically obtained as the phase-matched radiation of the experimental signal spectrum shown in Fig. 8.

ing both phase matching and stationarity. Note nevertheless that in some particular conditions, the overlap between OPA gain and wave mode curves can reach a large spectral extension, thus being able to support few cycle transform limited pulses.

We have completed the characterization of the amplified radiation by computing the near-field distribution in the $(r, t)$ space ( $r$ being the radial coordinate). In Fig. 10 we present as example the numerical result obtained again from the evaluation of the inverse Hankel-Fourier transform of the averaged experimental power spectrum of the visible signal radiation, with the assumption of cylindrical symmetry and flat spatial and temporal phases. The plot in the figure reveals an

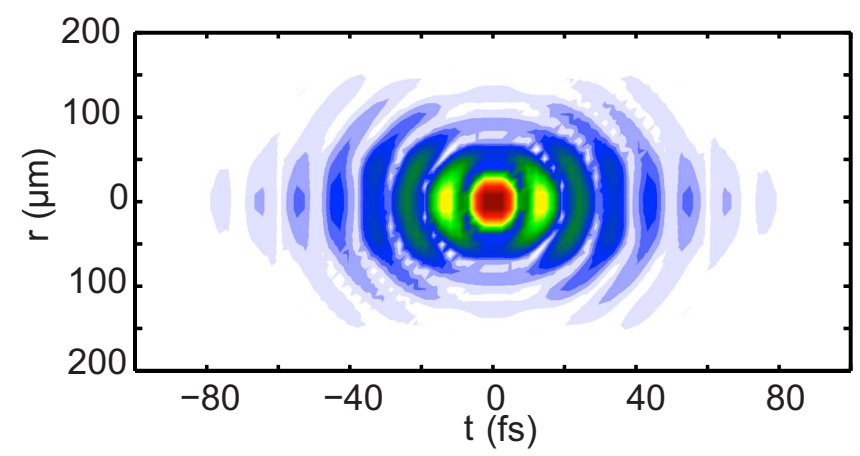

FIG. 10. (Color online) Numerical evaluation of the signal nearfield space-time intensity profile associated with the measured O-shaped spectrum of Fig. 1(b), under the assumption of flat spatial and temporal phases. 
onion-shaped structure with a central peak pulse localized within few micrometers and femtoseconds, respectively. It can be shown that the corresponding idler radiation possesses the same spatiotemporal localization properties.

\section{DISCUSSION AND CONCLUSIONS}

In this paper, we have proposed a technique for highenergy broadband conical wave packet generation and controlled amplification based on a continuum seeded OPA process. We have shown in different crystals and phasematching configurations that by appropriately focusing a spatially and spectrally broad coherent weak seed, it is possible in OPA to selectively amplify a phase-locked conical wave packet.

We have illustrated in particular the experimental generation and amplification of broadband X-type and O-type waves, respectively. We have seen, by analyzing the case of $\mathrm{X}$ waves, that the conical WPs supported by the nonlinear amplification process, and thus having both signal and idler contributing to the total amplified field, can be characterized along the propagation direction by a well-defined global phase velocity, and have subdispersive propagation properties in the nonlinear material. Even in the case of chirped pulse generation, we have found that, if the conjugation condition is respected, the phase velocity of the conical pulse can be defined as a nonlocal quantity, which moreover turns out to be equal to that of the pump pulse. It is interesting to note that the features highlighted so far for the phasematched radiation in OPA may be recognized in other wave packets, such as those described in Ref. [30] and named $\mathrm{Y}$ waves and for which further analysis is in progress.

The stationarity of the generated WPs has been demonstrated for a limited bandwidth since the stationarity requirement turns out to be equivalent to the phase-matching conditions only in the temporal quadratic approximation. As a consequence, we have shown that by suitably spectral windowing the supercontinuum input seed injected in the OPA, it is possible to generate stationary conical WPs, such as portions of $\mathrm{X}$ waves, or $\mathrm{O}$ waves, in normal and anomalous dispersion regimes, respectively. Moreover we have shown that the phase velocity of the $X$ wave modes generated in OPA can be directly extracted from the dispersion curve of the spatiotemporal spectra, which are formed by signal and idler contributions simultaneously. In addition, the tunability of the amplified field phase velocity, which is given by the phase velocity of the pump pulse, (and thus the ability to generate either subluminal or luminal WPs), is guaranteed by the possibility to tune the temperature or the phase-matching angle of the nonlinear crystal used (tuning of the OPA gain), and is certainly of great interest for the optimization of phase-matched related nonlinear problems. It is also worth mentioning in this context that the control of phase velocity is important to achieve the desired phase matching in other energy transfer applications, such as Extreme uv radiation generation, where OPA pulses will be used as pump.

Finally, it is interesting to note that the dispersion properties of particular conical waves which can be generated in OPA processes like for instance the $\mathrm{X}$ waves presented here, can be of great interest for applications. Indeed, we recall that the primary effects of angular dispersion are the tilting of the pulse front of the wave packet with respect to the wave front, and the effective group velocity dispersion that could be used as a linear mechanism of pulse compression [20,31]. This opens the way to future investigations based on the temporal tailoring of the input seed (chirped seed), aimingeven in the case where the generated WPs are not transform limited-at the possibility of obtaining pulses that selfcompress in vacuum from the OPA crystal output, without facing pump depletion effects inside the nonlinear medium. Work is in progress in this direction.

\section{ACKNOWLEDGMENTS}

The authors acknowledge support from Consorzio Nazionale Interuniversitario per le Scienze fisiche della Materia (CNISM), project INNESCO and thank Daniele Faccio for useful discussions. M.C. acknowledges support from MIUR, Project No. RBIN04NYLH. P.D.T. acknowledges support from the Marie Curie Chair project STELLA, Contract No. MEXC-CT-2005-025710, and from the Lithuanian Science and Technology foundation (ConTex project).
[1] J. Durnin, J. J. Miceli, and J. H. Eberly, Phys. Rev. Lett. 58, 1499 (1987).

[2] J. Lu and J. F. Greenleaf, IEEE Trans. Ultrason. Ferroelectr. Freq. Control 37, 438 (1990).

[3] P. Saari and K. Reivelt, Phys. Rev. Lett. 79, 4135 (1997).

[4] Z. Liu and D. Fan, J. Mod. Opt. 45, 17 (1998).

[5] C. Conti, P. Di Trapani, and S. Trillo, Top. Appl. Phys. 114, 433 (2009) and see references therein.

[6] E. Gaizauskas, A. Dubietis, V. Kudriasov, V. Sirutkaitis, A. Couairon, D. Faccio, and P. Di Trapani, Top. Appl. Phys. 114, 457 (2009) and see references therein.

[7] D. McGloin and K. Dholakia, Contemp. Phys. 46, 15 (2005).

[8] A. Averchi, D. Faccio, R. Berlasso, M. Kolesik, J. V. Moloney, A. Couairon, and P. Di Trapani, Phys. Rev. A 77, 021802(R)
(2008)

[9] P. Di Trapani, G. Valiulis, A. Piskarskas, O. Jedrkiewicz, J. Trull, C. Conti, and S. Trillo, Phys. Rev. Lett. 91, 093904 (2003).

[10] O. Jedrkiewicz, J. Trull, G. Valiulis, A. Piskarskas, C. Conti, S. Trillo, and P. Di Trapani, Phys. Rev. E 68, 026610 (2003).

[11] M. Kolesik, E. M. Wright, and J. V. Moloney, Phys. Rev. Lett. 92, 253901 (2004).

[12] D. Faccio, P. Di Trapani, S. Minardi, A. Bramati, F. Bragheri, C. Liberale, V. Degiorgio, A. Dubietis, and A. Matijosius, J. Opt. Soc. Am. B 22, 862 (2005).

[13] D. Faccio, A. Averchi, A. Dubietis, P. Polesana, A. Piskarskas, P. Di Trapani, and A. Couairon, Opt. Lett. 32, 184 (2007).

[14] D. Faccio, A. Averchi, A. Couairon, M. Kolesik, J. V. Molo- 
ney, A. Dubietis, G. Tamosauskas, P. Polesana, A. Piskarskas, and P. Di Trapani, Opt. Express 15, 13077 (2007).

[15] V. P. Koronkevich, I. A. Mikhaltsova, E. G. Churin, and Yu. I. Yurlov, Appl. Opt. 34, 5761 (1995).

[16] A. Vasara, J. Turunen, and A. T. Friberg, J. Opt. Soc. Am. A 6, 1748 (1989).

[17] S. Orlov, A. Piskarskas, and A. Stabinis, Opt. Lett. 27, 2103 (2002).

[18] S. Longhi, Phys. Rev. E 69, 016606 (2004).

[19] R. Butkus, S. Orlov, A. Piskarskas, V. Smilgevicius, and A. Stabinis, Opt. Commun. 244, 411 (2005).

[20] G. Valiulis, A. Dubietis, and A. Piskarskas, Phys. Rev. A 77, 043824 (2008).

[21] M. A. Porras and P. Di Trapani, Phys. Rev. E 69, 066606 (2004).

[22] M. A. Porras, P. Di Trapani, and W. Hu, in Localized Waves, edited by H. E. Hernandes-Figueroa, M. Zamboni-Rached, and E. Recami (Wiley-Interscience, New York, 2007), Chap. 8, p. 217.

[23] O. Jedrkiewicz, A. Picozzi, M. Clerici, D. Faccio, and P. Di Trapani, Phys. Rev. Lett. 97, 243903 (2006).

[24] O. Jedrkiewicz, M. Clerici, A. Picozzi, D. Faccio, and P. Di Trapani, Phys. Rev. A 76, 033823 (2007).

[25] P. Di Trapani, G. Valiulis, W. Chinaglia, and A. Andreoni, Phys. Rev. Lett. 80, 265 (1998).

[26] S. Orlov, A. Stabinis, V. Smilgevicius, G. Valiulis, and A. Piskarskas, Opt. Lett. 32, 68 (2007).

[27] M. Clerici, O. Jedrkiewicz, E. Rubino, D. Faccio, L. Tartara, V. Degiorgio, and P. Di Trapani, Opt. Lett. 33, 2296 (2008).

[28] D. Faccio, A. Averchi, A. Lotti, M. Kolesik, J. V. Moloney, A. Couairon, and P. Di Trapani, Phys. Rev. A 78, 033825 (2008).

[29] H. Sonajalg and P. Saari, Opt. Lett. 21, 1162 (1996).

[30] M. A. Porras and A. Parola, Opt. Commun. 282, 644 (2009).

[31] L. Berge, S. Skupin, and G. Steinmeyer, Phys. Rev. A 79, 033838 (2009). 